\title{
Commissioning of the spede spectrometer with stable beams
}

\author{
Cox, D. M.
}

2017-03

Cox , D M , Pakarinen , J , Papadakis , P , Butler , P A , Greenlees , P T , Konki , J , Herzberg , R -D , O'Neill , G G \& Rahkila , P 2017 , ' Commissioning of the spede spectrometer with stable beams ' , Acta Physica Polonica. B , vol. 48 , no. 3 , pp. 403-408 . https://doi.org/10.5506/A

http://hdl.handle.net/10138/189713

https://doi.org/10.5506/APhysPolB.48.403

cc_by_nc

publishedVersion

Downloaded from Helda, University of Helsinki institutional repository.

This is an electronic reprint of the original article.

This reprint may differ from the original in pagination and typographic detail.

Please cite the original version. 


\title{
COMMISSIONING OF THE SPEDE SPECTROMETER WITH STABLE BEAMS*
}

\author{
D.M. Cox ${ }^{a, b}$, J. PAKARINEn ${ }^{a, b}$, P. PAPADAKis ${ }^{a, b}$, P.A. Butler ${ }^{c}$ \\ P.T. Greenlees ${ }^{a, b}$, J. Konki ${ }^{a, b}$, R.-D. HerzBerG ${ }^{\mathrm{c}}$ \\ G.G. O'NEILL ${ }^{\mathrm{c}}$, P. RAHKILA ${ }^{\mathrm{a}, \mathrm{b}}$ \\ ${ }^{a}$ University of Jyväskylä, Department of Physics \\ P.O. Box 35, 40014 University of Jyväskylä, Finland \\ ${ }^{\mathrm{b}}$ Helsinki Institute of Physics, 00014, Helsinki, Finland \\ ${ }^{\mathrm{c}}$ Oliver Lodge Laboratory, University of Liverpool \\ Liverpool, L69 7ZE, United Kingdom
}

(Received December 14, 2016)

The SPectrometer for Electron DEtection (SPEDE) has been constructed for in-beam nuclear structure studies using radioactive ion beams. SPEDE employs a silicon detector for detecting conversion electrons. It is designed to be used in conjunction with the MINIBALL spectrometer at HIE-ISOLDE, CERN.

DOI:10.5506/APhysPolB.48.403

\section{Introduction}

Gamma-ray spectroscopy has long been a favoured tool to study nuclear structure. In recent years, arrays such as MINIBALL [1] have been employed with radioactive ion beams (RIBs) to allow ever more exotic nuclei to be studied. For many regions of the nuclear chart, however, internal conversion can compete or even dominate over $\gamma$-ray emission. For this reason, a number of electron spectrometers have been developed over the years, SACRED [2] for instance. More recently, electron spectrometers have been developed that can by employed simultaneously with $\gamma$-ray spectrometers, an example of this is the SAGE spectrometer [3] at the Accelerator Laboratory of the Physics Department of the University of Jyväskylä (JYFL).

\section{Description of SPEDE}

SPEDE $[4,5]$ is designed to measure conversion electrons emitted from excited states in nuclei populated in Coulomb-excitation reactions. It will

* Presented at the Zakopane Conference on Nuclear Physics "Extremes of the Nuclear Landscape", Zakopane, Poland, August 28-September 4, 2016. 
be employed in conjunction with the MINIBALL array at HIE-ISOLDE, CERN. The scientific motivation for SPEDE can be found in a number of accepted proposals [6-9].

Figure 1 shows a graphical representation of SPEDE. The SPEDE silicon detector can be seen to the left (in backwards angle with respect to the beam). An absorber foil in front of the detector is used for reducing the flux of low-energy $\delta$ electrons onto the detector. The choice of geometry also minimises the $\delta$-electron flux as they are strongly forward focused.

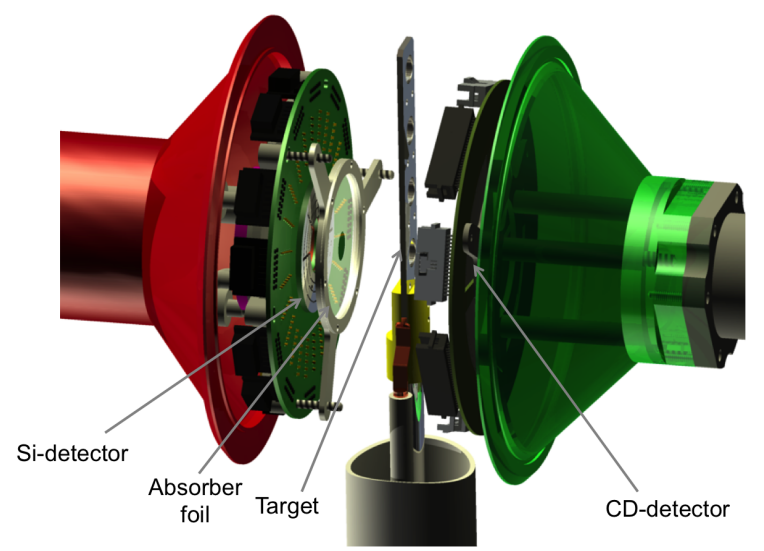

Fig. 1. Schematic of SPEDE with key components labelled. Beam enters from left. Part of the target chamber has been removed for visualization purposes.

The geometry of the SPEDE silicon detector is explained in detail by Konki et al. [4]. The detector is $0.5 \mathrm{~mm}$ thick and is segmented into 24 pixels. The inner radius of the detector is $17 \mathrm{~mm}$ allowing for the beam to pass through. The outer radius of the detector is $44.6 \mathrm{~mm}$.

The CD detector [10] is used to detect the scattered target- and projectilelike particles downstream from the target. The position sensitivity of the CD, MINIBALL and SPEDE allows for Doppler correction to be performed for both $\gamma$ rays and conversion electrons. The target is mounted on a target ladder which can accommodate four different targets. Up to $+5 \mathrm{kV}$ high voltage can be applied to the target to further suppress the possible $\delta$-electron background. The target position in use can be changed without breaking the vacuum.

\section{Performance}

\subsection{Offline performance - source tests}

The offline performance of SPEDE was tested using an open ${ }^{133} \mathrm{Ba}$ calibration source. The energy resolution (FWHM) of the SPEDE silicon detector without an absorber foil was $6.0 \mathrm{keV}$ at $320 \mathrm{keV}$. The addition of a $12 \mu \mathrm{m}$ 
aluminized mylar foil in front of the detector slightly degrades the resolution to $\sim 7.5 \mathrm{keV}$ at $320 \mathrm{keV}$. Figure 2 highlights both the slight degradation in resolution along with the shift of the detected energy, where the $320 \mathrm{keV}$ peak has been shifted down by $\sim 3 \mathrm{keV}$. It should also be noted that these spectra are normalised to the counts in the $320 \mathrm{keV}$ peak. It is clear that the foil has a much greater effect on transmission for lower energy electrons, the peak at $45 \mathrm{keV}$ has $\sim 50 \%$ less counts after normalisation. Figure 2 also shows the effect of applying a voltage to the target. As expected, the measured energy is shifted downwards by an amount equivalent to the voltage applied, e.g. $320 \mathrm{keV} \rightarrow 315 \mathrm{keV}$.

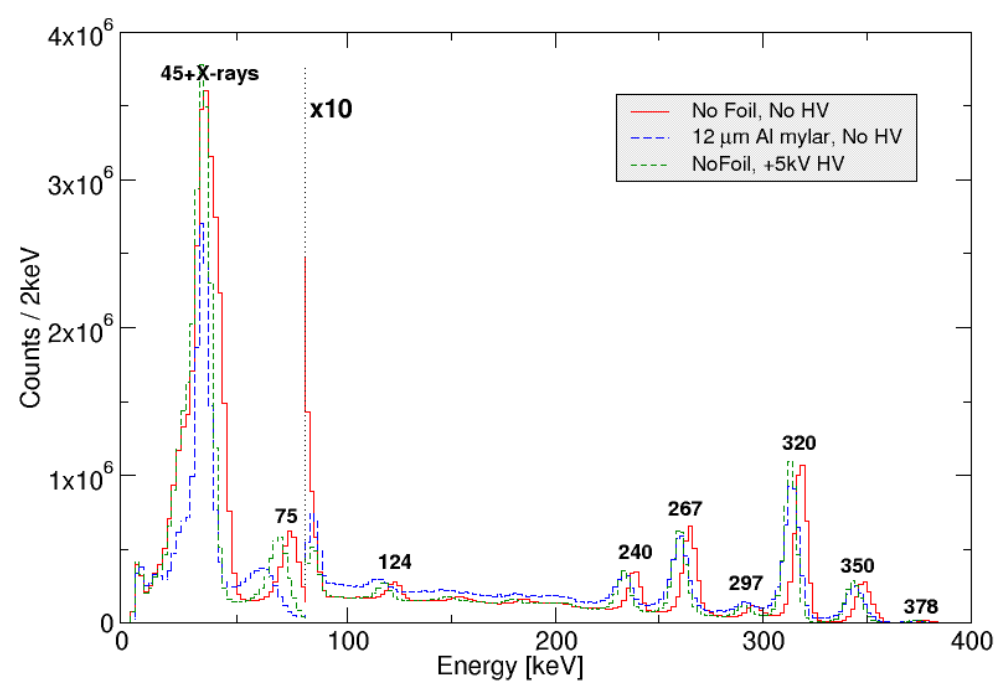

Fig. 2. Effect of the absorber foil and HV on count rate, measured energy and detector resolution (normalised to $320 \mathrm{keV}$ peak).

\subsection{Online performance at JYFL}

\subsubsection{SIMO — SIlicon MOnitor}

The SIlicon MOnitor (SIMO) was developed to allow for detection of scattered particles in the in-beam tests. SIMO consists of six $1 \mathrm{~cm}^{2}$ PINdiodes (PINs) arranged at a distance of $29.2 \mathrm{~mm}$ downstream from the target and at radial distance of $15.2 \mathrm{~mm}$ from the beam axis. The angle between two PINs was $30^{\circ}$. The angular coverage of SIMO is between $19^{\circ}$ and $35^{\circ}$. A Phase I-type germanium detector was used in the in-beam test to allow for the detection of $\gamma$-rays and for $\gamma$-electron cross-coincidence measurements.

In order to characterize the performance of SPEDE, the Coulomb-excitation reaction of a ${ }^{82} \mathrm{Kr}$ beam on ${ }^{197} \mathrm{Au}$ target was selected. ${ }^{197} \mathrm{Au}$ has a wellknown level scheme with a number of highly converted transitions [11]. The 
beam energy used was $4.26 \mathrm{MeV} / u$. An advantage of the chosen reaction is that the target- and projectile-like particles are well-separated in energy. The inset of Fig. 3 shows a partial level scheme for ${ }^{197} \mathrm{Au}$. Table I shows a number of transitions seen in ${ }^{197} \mathrm{Au}$ along with emitted $\gamma$-ray, K-, L- and M-conversion electron energies.

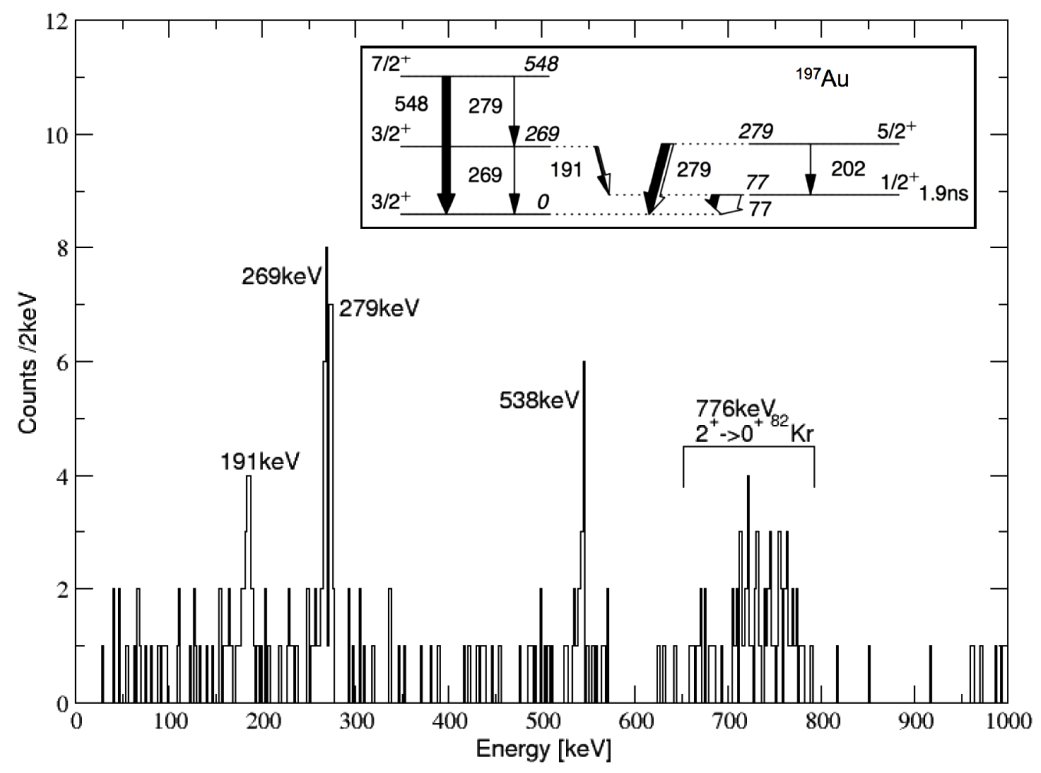

Fig. 3. Particle gated Doppler corrected $\gamma$-ray energy spectrum, for target-like particles in the reaction ${ }^{82} \mathrm{Kr}\left({ }^{197} \mathrm{Au},{ }^{197} \mathrm{Au}^{*}\right)$. Prominent peaks have been labelled. Partial level scheme for ${ }^{197} \mathrm{Au}$ is shown in the inset.

TABLE I

Transition energies in ${ }^{197} \mathrm{Au}$ with their corresponding K-, L- and M-conversion electron energies. Bracketed values are detected energies owing to high voltage applied to the target.

\begin{tabular}{c|cccc}
\hline \hline Transition & $\begin{array}{c}\gamma \text { ray } \\
{[\mathrm{keV}]}\end{array}$ & $\begin{array}{c}\mathrm{K}-e^{-} \\
{[\mathrm{keV}]}\end{array}$ & $\begin{array}{c}\mathrm{L}-e^{-} \\
{[\mathrm{keV}]}\end{array}$ & $\begin{array}{c}\mathrm{M}-e^{-} \\
{[\mathrm{keV}]}\end{array}$ \\
\hline$\frac{3}{2}^{+} \rightarrow \frac{1}{2}^{+}$ & 191 & $110(105)$ & $178(173)$ & $188(183)$ \\
$\frac{3}{2}^{+} \rightarrow \frac{3}{2}^{+}$ & 269 & $188(183)$ & $256(251)$ & $266(261)$ \\
$\frac{7}{2}^{+} \rightarrow \frac{3}{2}^{+}$and $\frac{5}{2}^{+} \rightarrow \frac{3}{2}^{+}$ & 279 & $198(193)$ & $266(261)$ & $276(271)$
\end{tabular}


Using an event in SIMO as the trigger condition and measuring the detected $\gamma$ rays and electrons in coincidence allows one to disentangle the transitions of interest from the overwhelming background. To further enhance the selectivity, the accepted energy range of the recoiling particle was selected to be within the range expected for target-like particles.

In Fig. 3, a number of $\gamma$ rays associated with transitions in ${ }^{197} \mathrm{Au}$ are visible. These are Doppler corrected according to the energy of the recoiling ${ }^{197} \mathrm{Au}$ particles. The $2_{1}^{+} \rightarrow 0_{1}^{+}$transition in ${ }^{82} \mathrm{Kr}$ can also be seen, but it has been effectively smeared out due to the Doppler broadening.

Figure 4 shows the corresponding Doppler-corrected conversion-electron energy spectrum. It should be noted that this measurement was performed with $+5 \mathrm{kV}$ applied to the target with no foil. The measured FWHM after Doppler correction is $\sim 10 \mathrm{keV}$. Annotated in the figure are a number of K-, L- and M-conversion electron peaks. Even with this relatively simple case, the challenge of electron spectroscopy is highlighted with K-, L- and M-conversion peaks from different transitions overlapping. Conversely, this added complexity brings added opportunities for studying nuclei. Of special interest are the E0 transitions common in the Pb-region $[12,13]$ which can only be observed by employing conversion-electron spectroscopy.

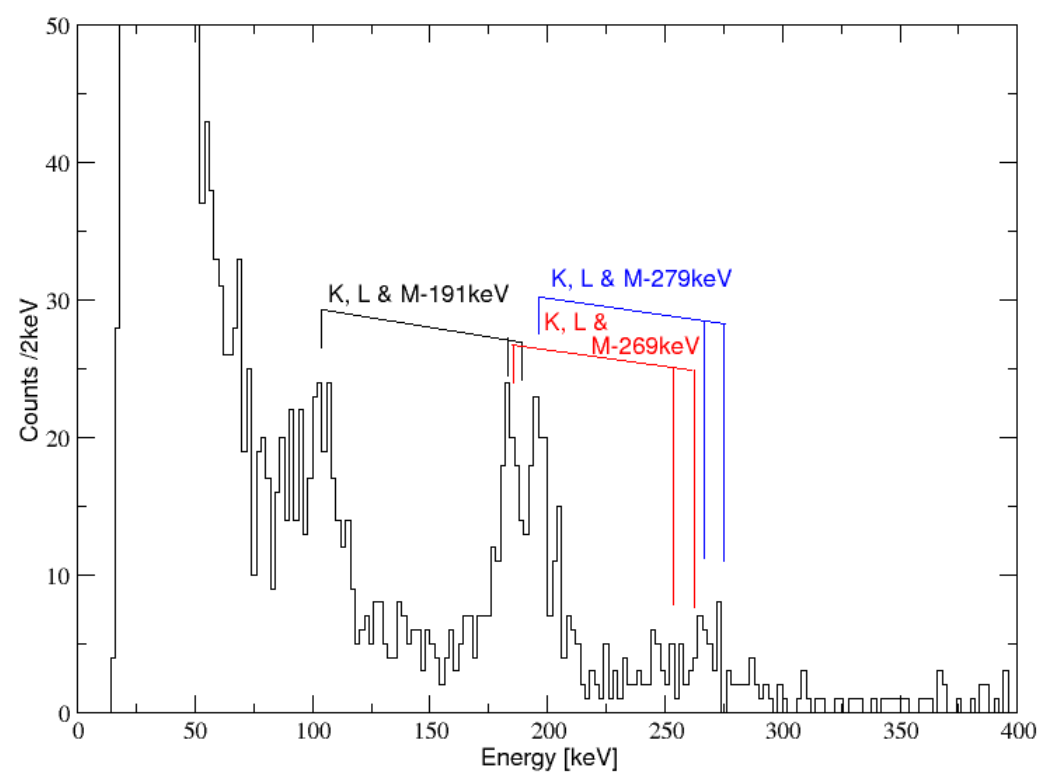

Fig. 4. Particle gated Doppler corrected conversion-electron spectrum, for targetlike particles in the reaction ${ }^{82} \mathrm{Kr}\left({ }^{197} \mathrm{Au},{ }^{197} \mathrm{Au}^{*}\right)$. Conversion-electron lines and related transition energies have been marked. 


\section{Summary}

SPEDE has been successfully commissioned in-beam at JYFL using the reaction ${ }^{82} \mathrm{Kr}\left({ }^{197} \mathrm{Au},{ }^{197} \mathrm{Au}^{*}\right)$. Recoil gating and applying Doppler correction was shown to be viable in identifying transitions of interest.

This work is supported by the Marie Curie Career Integration Grant (grant No. 304033) and by the Academy of Finland (grant No. 257562). Funding is gratefully acknowledged from the United Kingdom Science and Technology Facilities Council (UK STFC). The GAMMAPOOL network is acknowledged.

\section{REFERENCES}

[1] N. Warr et al., Eur. Phys. J. A 49, 1 (2013).

[2] P.A. Butler et al., Nucl. Instrum. Methods Phys. Res. A 381, 433 (1996).

[3] J. Pakarinen et al., Eur. Phys. J. A 50, 53 (2014).

[4] J. Konki et al., EPJ Web Conf. 63, 01019 (2013).

[5] P. Papadakis et al., JPS Conf. Proc. 6, 030023 (2015).

[6] J. Pakarinen et al., Probing Intruder Configurations in ${ }^{186,188} \mathrm{~Pb}$ using Coulomb Excitation, CERN-INTC, 004:370, 2013.

[7] K. Wrzosek-Lipska et al., Coulomb Excitation of ${ }^{182-184} \mathrm{Hg}$ : Shape Coexistence in the Neutron-deficient Lead Region, CERN-INTC, 063:364, 2012.

[8] P.A. Butler et al., Addendum (IS475) to the ISOLDE and Neutron Time-of-Flight Committee Measurements of Octupole Collectivity in Odd-mass Rn and Ra Nuclei Using Coulomb Excitation, CERN-INTC, 008:244-ADD-1, 2011.

[9] P.A. Butler et al., Measurement of Octupole Collectivity in RN and Ra Nuclei Using Coulomb Excitation, CERN-INTC, 019:347-ADD-1, 2013.

[10] A.N. Ostrowski et al., Nucl. Instrum. Methods Phys. Res. A 480, 448 (2002).

[11] F.K. McGowan, W.T. Milner, R.L. Robinson, P.H. Stelson, Ann. Phys. 63, 549 (1971).

[12] G.D. Dracoulis et al., Phys. Rev. C 69, 054318 (2004).

[13] J. Pakarinen et al., Phys. Rev. C 75, 014302 (2007). 